\title{
First clinical experience with a dedicated MRI-guided high-intensity focused ultrasound system for breast cancer ablation
}

\author{
Laura G. Merckel ${ }^{1}$ - Floor M. Knuttel ${ }^{1} \cdot$ Roel Deckers $^{2} \cdot$ Thijs van Dalen $^{3}$. \\ Gerald Schubert $^{4}$ • Nicky H. G. M. Peters ${ }^{1}$ - Teun Weits ${ }^{5}$ • Paul J. van Diest ${ }^{6}$. \\ Willem P. Th. M. Mali ${ }^{1}$ Paul H. H. B. Vaessen ${ }^{7}$ Joost M. H. H. van Gorp ${ }^{8}$ • \\ Chrit T. W. Moonen ${ }^{2} \cdot$ Lambertus W. Bartels $^{2} \cdot$ Maurice A. A. J. van den Bosch ${ }^{1}$
}

Received: 16 October 2015 /Revised: 12 December 2015 / Accepted: 15 January 2016 /Published online: 6 February 2016

(C) The Author(s) 2016. This article is published with open access at Springerlink.com

\begin{abstract}
Objectives To assess the safety and feasibility of MRI-guided high-intensity focused ultrasound (MR-HIFU) ablation in breast cancer patients using a dedicated breast platform.

Methods Patients with early-stage invasive breast cancer underwent partial tumour ablation prior to surgical resection. MR-HIFU ablation was performed using proton resonance frequency shift MR thermometry and an MR-HIFU system specifically designed for breast tumour ablation. The presence and extent of tumour necrosis was assessed by histopathological analysis of the surgical specimen. Pearson correlation coefficients were calculated to assess the relationship between
\end{abstract}

Laura G. Merckel

L.G.Merckel-2@umcutrecht.nl

1 Department of Radiology, University Medical Center Utrecht, HP E 01.132, P.O. Box 85500, 3584 CX Utrecht, The Netherlands

2 Image Sciences Institute, University Medical Center Utrecht, Utrecht, The Netherlands

3 Department of Surgery, Diakonessenhuis Utrecht, Utrecht, The Netherlands

4 Philips Healthcare, Best, The Netherlands

5 Department of Radiology, Diakonessenhuis Utrecht, Utrecht, The Netherlands

6 Department of Pathology, University Medical Center Utrecht, Utrecht, The Netherlands

7 Department of Anesthesiology, University Medical Center Utrecht, Utrecht, The Netherlands

8 Department of Pathology, Diakonessenhuis Utrecht, Utrecht, The Netherlands sonication parameters, temperature increase and size of tumour necrosis at histopathology.

Results Ten female patients underwent MR-HIFU treatment. No skin redness or burns were observed in any of the patients. No correlation was found between the applied energy and the temperature increase. In six patients, tumour necrosis was observed with a maximum diameter of 3-11 $\mathrm{mm}$. In these patients, the number of targeted locations was equal to the number of areas with tumour necrosis. A good correlation was found between the applied energy and the size of tumour necrosis at histopathology (Pearson $=0.76, \mathrm{p}=0.002)$.

Conclusions Our results show that MR-HIFU ablation with the dedicated breast system is safe and results in histopathologically proven tumour necrosis.

Key Points

- MR-HIFU ablation with the dedicated breast system is safe and feasible

- In none of the patients was skin redness or burns observed

- No correlation was found between the applied energy and the temperature increase

- The correlation between applied energy and size of tumour necrosis was good

Keywords High-intensity focused ultrasound · Ablation · Breast cancer $\cdot$ Magnetic resonance imaging $\cdot$ Minimally invasive treatment

\section{Introduction}

Breast cancer is the most common malignancy among women worldwide [1]. The disease is currently frequently diagnosed at an early stage because of 
mammographic screening programmes and improved awareness [2]. Over the past decades, breast cancer treatment has evolved towards less invasive local treatment. Breast-conserving therapy (BCT), i.e. lumpectomy with additional radiotherapy, is currently standard-ofcare in patients with early-stage breast cancer and has shown equal survival rates compared to radical mastectomy [3, 4]. A range of minimally invasive techniques holds promise for replacing lumpectomy by local breast tumour ablation, for example cryoablation, radiofrequency ablation or microwave ablation. All these techniques, however, require percutaneous insertion of a probe into the breast tumour [5-7]. High-intensity focused ultrasound (HIFU) is a completely noninvasive technique that can be used for thermal ablation in a target volume deep within the body [8]. Imaging during minimally invasive treatment is crucial to localize the target area and monitor the treatment procedure. Magnetic resonance imaging (MRI) offers excellent anatomical imaging for treatment planning by defining the target volume and organs at risk, is able to provide real-time temperature monitoring during therapy, and allows direct evaluation of treatment results [9-11]. In 2001, Huber et al. [12] described the first MRI-guided HIFU (MR-HIFU) treatment in a breast cancer patient. Subsequently several groups reported on MR-HIFU ablation of malignant breast tumours prior to surgical resection [13-16]. Overall, authors concluded that MR-HIFU ablation of breast cancer was technically feasible. Complete tumour necrosis, however, was achieved in only $20-50 \%$ of patients, whereas complete tumour ablation has to be ensured before surgical resection can be omitted. Optimizing these results is necessary for MR-HIFU treatment to be considered as a clinically attractive alternative to surgery for local breast tumour control.

In this study, we report the first experiences on tumour ablation in breast cancer patients using an MRHIFU breast platform specifically designed for breast tumour ablation [17]. In previous studies, treatments were performed using MR-HIFU systems with a single transducer targeting the breast from the anterior using a point-by-point ablation method [13, 15]. In contrast, with our dedicated platform, the breast is targeted laterally, consequently reducing the risk of unintended heating of the heart and lungs. In addition, the wide transducer aperture decreases the local energy density on the skin during ablation. Furthermore, a volumetric ablation approach is used, resulting in larger and more homogeneous ablation volumes and a reduction in treatment duration $[18,19]$. The aim of the current study was to assess the safety and feasibility of tumour ablation in breast cancer patients using the dedicated breast platform.

\section{Materials and methods}

\section{Patients}

The study protocol was approved by the institutional review board of the University Medical Center Utrecht and written informed consent was obtained from all patients. Patients were recruited in the Diakonessenhuis Utrecht and included in the University Medical Center Utrecht between September 2012 and June 2014. Inclusion criteria were: women aged $>18$ years; World Health Organization (WHO) performance status $\leq 2$; body weight $\leq 80 \mathrm{~kg}$; clinically staged $\mathrm{T} 1$ 2 , histopathologically proven invasive breast cancer. Exclusion criteria were: neoadjuvant systemic therapy; contraindications for MRI; macro-calcifications; scar tissue or surgical clips in the direct path of the ultrasound beams.

All patients underwent an MRI examination on a 3-T clinical MR scanner (Achieva, Philips Healthcare, Best, The Netherlands) to assess whether the following additional inclusion criteria were met: maximum tumour diameter $\geq 1.0 \mathrm{~cm}$; tumour location within the reach of the HIFU transducers with the patient in prone position; distance from skin and pectoral muscle to the centre of the target $\geq 1.0 \mathrm{~cm}$.

\section{Dedicated MR-HIFU breast platform}

MR-HIFU ablation was performed using a dedicated MRHIFU breast platform (Sonalleve-based prototype, Philips Healthcare, Vantaa, Finland) which was integrated into a 1.5-T MR scanner (Achieva, Philips Healthcare, Best, The Netherlands). During MR-HIFU treatment, patients were placed in prone position on the HIFU table top with the targeted breast in the water-filled breast cup surrounded by eight separate 32-element transducers distributed over a $270^{\circ}$ circular arc. The specifications of the system have been previously described in more detail [17]. In addition, Deckers et al. [20] recently published a performance analysis of the breast platform.

\section{MR-HIFU treatment}

\section{Procedural sedation}

Patients were under procedural sedation during MR-HIFU treatment. A team of procedural sedation and analgesia specialists monitored the cardiorespiratory functions and administered sedative agents and analgesics intravenously. In the first two patients, procedural sedation was maintained using continuous propofol infusion and an additional opioid analgesic prior to each sonication. Due to undesired patient motion and variations in the breathing pattern during these first two 
treatments, a combination of propofol and esketamine was used during all other treatments.

\section{MR imaging}

Figure 1 shows a schematic overview of the procedures and MR pulse sequences during treatment. Treatment planning was performed based on the localization of the breast tumour on dynamic contrast-enhanced (DCE) MR imaging (dynamic scan time $78.3 \mathrm{~s}$; TR/TE $6.6 / 3.2 \mathrm{~ms}$; flip angle $10^{\circ}$; turbofactor $=36$; acquisition voxel size $1.12 \times 1.12 \times 2.0 \mathrm{~mm}^{3} ; 140$ slices; matrix size $304 \times 180$; SPIR fat suppression). One dynamic was acquired before and four dynamics were acquired directly after injection of a gadolinium-based contrast agent (GBCA) (0.1 ml gadobutrol/kg body weight (Gadovist, Bayer Schering Pharma AG, Berlin, Germany)). Because of the potential hazard of heating a GBCA inside the body, a waiting time of 30 min was maintained between contrast injection and the first sonication. Before each sonication, a short T1weighted scan was performed, which was visually compared to T1-weighted images acquired at the beginning of the treatment procedure to confirm accurate patient positioning.

During sonications, subtraction-based PRFS (proton resonance frequency shift) MR thermometry using an echo planar imaging (EPI) pulse sequence was performed with the following parameters: TR/TE 70/30 ms; flip angle 20 ; EPI-factor 23 ; acquisition voxel size $1.67 \times 1.67 \times 5.0 \mathrm{~mm}^{3}$; 4 slices; matrix size $96 \times 92$; composite RF pulse fat suppression. Four planes were monitored with a temporal resolution of $2.25 \mathrm{~s}$ : a coronal and sagittal slice through the focal point, a coronal

Fig. 1 A schematic overview of procedures during MRI-guided high-intensity focused ultrasound (MR-HIFU) treatment near-field slice positioned $9.5 \mathrm{~mm}$ anterior to the focal point, and a far-field slice manually positioned at the pectoral muscle. A look-up-table (LUT)-based correction method was used to correct errors in the MR temperature maps caused by respiration-induced magnetic field disturbances [20, 21]. Relative temperature maps were calculated on the fly and overlaid onto T1-weighted, fat-suppressed magnitude images of the thermometry sequence. In patients three to ten, $160 \mathrm{mg} /$ $\mathrm{L} \mathrm{MnCl}_{2} \cdot 4 \mathrm{H}_{2} \mathrm{O}$ was added to the water in the breast cup to shorten its $\mathrm{T} 2$ signal and prevent ghosting artefacts during MR thermometry due to possible subtle motion of the water in the breast cup. The same DCE-MR scan that was used for treatment planning was repeated directly after MR-HIFU ablation for treatment evaluation.

\section{High-intensity focused ultrasound ablation}

In this first study with the MR-HIFU breast platform, partial tumour ablation was performed to be able to analyze the location and size of separate sonications and to assess the relationship between different sonication parameters and the size of tumour necrosis at histopathology. Low energy test sonications were performed prior to therapeutic sonications to verify the focal spot position. A correction was performed in case of spatial misalignment. Test sonications were 3-mm treatment cells with low (20-40 W) acoustic power. Therapeutic sonications were performed using a volumetric ablation technique with concentric circular trajectories of increasing size [18]. The resulting treatment cells, i.e. the differently sized ablation volumes, had an ellipsoidal shape with nominal diameters of

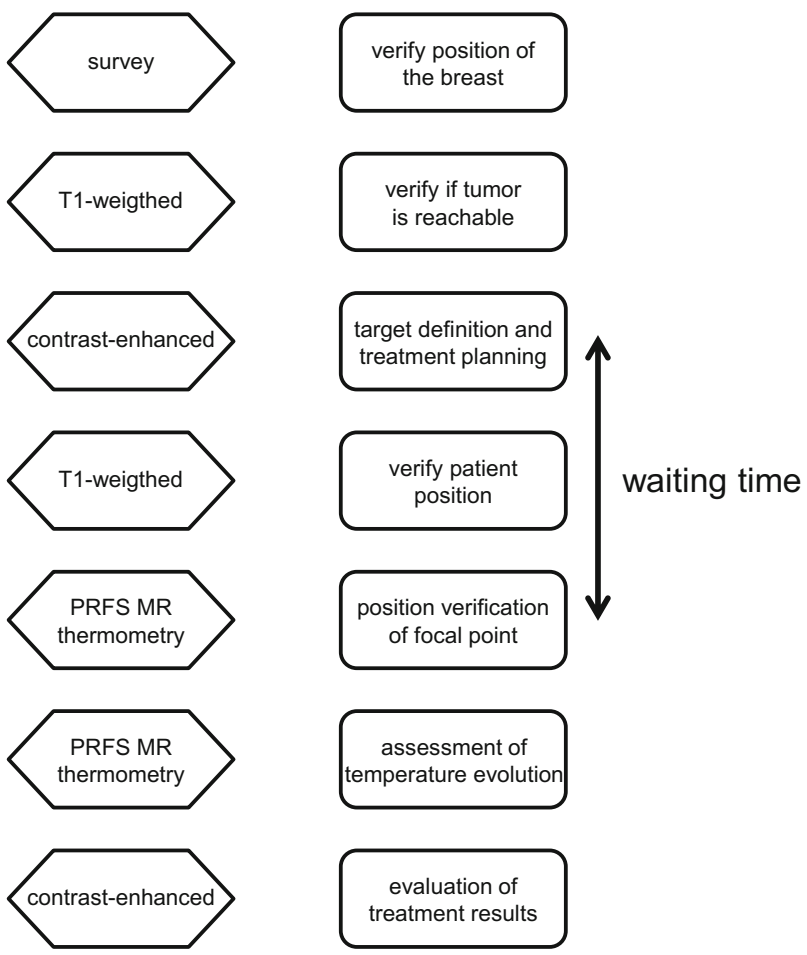

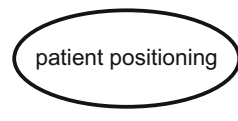
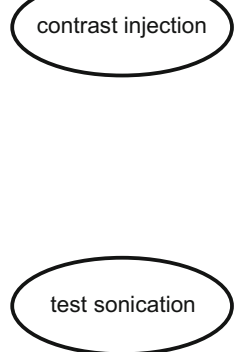

therapeutic sonication 
$3 \times 2 \times 2 \mathrm{~mm}^{3}$ or $6 \times 4 \times 4 \mathrm{~mm}^{3}$ (size of the volume bound by the iso-intensity surface at $-6 \mathrm{~dB}$ of the peak value in the centre) and a sonication duration of 20 and $24.5 \mathrm{~s}$, respectively. The applied acoustic power during therapeutic sonications varied between 50 and $100 \mathrm{~W}$ with a frequency of $1.45 \mathrm{MHz}$. Multiple sonications were allowed at one or more locations within tumours. Each sonication was followed by a period of cooling. Sonications were aborted when temperatures $\geq 80^{\circ} \mathrm{C}$ were observed in the MR temperature maps. Note that such apparent temperature elevations are not necessarily real, since the occurrence of artefacts (due to breathing or patient motion) may corrupt temperature measurements and lead to erroneous observation of excessive temperatures.

\section{After MR-HIFU treatment}

After MR-HIFU treatment, patients were admitted to a clinical ward for a minimum of $3 \mathrm{~h}$ to ensure stable haemodynamic function. Surgery was performed within $48 \mathrm{~h}$ and 10 days after MR-HIFU treatment. Clinical management of the axilla was performed according to standard clinical guidelines by a sentinel lymph node biopsy (SLNB) or axillary lymph node dissection. After surgical resection, tissue was submerged in formalin. The excised tissue containing the tumour was dissected into slices of approximately $5 \mathrm{~mm}$. Microscopic sections of $4 \mu \mathrm{m}$ were cut and stained with haematoxylin and eosin (H\&E) for histological analysis.

\section{Safety and feasibility}

After MR-HIFU treatment, the skin of the treated breast was evaluated by a physician for the presence of skin burns or redness. Patients were asked to report pain scores according to the numerical rating scale, with a score of 0 (no pain) to 10 (worst pain imaginable) [22]. Monitoring of adverse events was done until surgery. A radiologist compared the DCEMRI before and after MR-HIFU ablation to assess the presence of non-perfused volumes (NPVs) after ablation. For each sonication, the maximum temperature was reported based on the median temperature evolution in nine pixels in the centre of mass of the heating at the end of sonications. In addition, the maximum diameter of the area that reached a temperature higher than $55^{\circ} \mathrm{C}$ was reported. All analyses were done for the coronal MR thermometry slice using software developed in Matlab (Mathworks, Natick, MA, USA).

For all performed sonications, the relationship between the duration and power of sonications and the temperature increase from baseline temperature as measured by MR thermometry was assessed using simple linear regression analyses. In addition, the correlation between duration, applied powers, temperature increase and the size of tumour necrosis at histopathology was investigated for each sonicated location. A Pearson correlation coefficient $(r)<0.25$ was considered to indicate a trivial correlation, between 0.25 and 0.5 a low correlation, between 0.51 and 0.75 a medium correlation, and $>0.76$ a high correlation. A $p$-value $\leq 0.05$ was considered to be significant.

A dedicated breast pathologist evaluated the presence and the size of the areas with tumour necrosis, which were manually delineated using Aperio ImageScope (Leica Microsystems, Rijswijk, The Netherlands).

\section{Results}

\section{Patients}

Seventeen patients were initially enrolled in the study. In five patients, an additional lesion was detected at pre-treatment MRI. Two of these patients were excluded due to logistical reasons because of additional diagnostic work-up, and three patients withdrew from the study themselves. In addition, two patients withdrew from the study because of fear of an epileptic insult during MR-HIFU treatment $(n=1)$ and claustrophobia $(n=1)$. Finally, ten patients underwent MR-HIFU treatment. Table 1 lists the demographic data of these patients.

\section{MR-HIFU treatment}

The overall duration of MR-HIFU treatment was on average $145 \mathrm{~min}$. The actual sonication time was $1.7 \mathrm{~min}$ (Table 2). An overview of the performed sonications per individual patient is provided in Table 3 . In the first and third patients, only one therapeutic sonication was performed. These were both aborted in an early phase due to the erroneous measurement of excessive temperatures caused by patient motion or a change in the breathing pattern. In the second patient, three of four therapeutic sonications were prematurely aborted (at $60.8 \%, 90.2 \%$ and $98.5 \%$ of the full sonication length) for the same reasons. In patients four to ten, 23 of $24(95.8 \%)$ therapeutic sonications were fully executed.

\section{Safety}

No skin redness or burns were observed in any of the patients. Patient seven developed three small white lumps with a maximum diameter of $0.5-1.5 \mathrm{~cm}$ on the skin of the treated breast in the days after MR-HIFU treatment. Histopathological analysis of a biopsy from one of these lumps showed no signs of abnormal tissue. Over time, the lumps resolved without intervention. Other minor adverse events were nausea and vomiting (in two patients) in the hours after treatment, probably related to the administered anaesthetics. After MR-HIFU treatment, eight patients reported no pain. The other two patients reported a pain score of 4 and 5, respectively. 
Table 1 Baseline characteristics of breast cancer patients who underwent MRI-guided high-intensity focused ultrasound (MR-HIFU) treatment

\begin{tabular}{ll}
\hline Patients & $\mathrm{n}(\%)$ \\
\hline No. of patients & 10 \\
Age in years, mean \pm SD & $54.8 \pm 12.5$ \\
Treated tumours & \\
$\quad$ Tumours in right breast & $6(60.0)$ \\
Tumour location & \\
$\quad$ Upper outer quadrant & $3(30.0)$ \\
Lower outer quadrant & $5(50.0)$ \\
Upper inner quadrant & $2(20.0)$ \\
Lower inner quadrant & $0(0.0)$ \\
Interval between HIFU and surgery & \\
$\quad$ Time in days, mean \pm SD & $5.0 \pm 2.2$ \\
Type of surgery & \\
Lumpectomy & $8(80.0)$ \\
Mastectomy & $1(10.0)$ \\
No surgery & $1(10.0)$ \\
Axilla & \\
Sentinel lymph node procedure & $8(80.0)$ \\
Axillary dissection & $1(10.0)$ \\
No axillary procedure & $1(10.0)$ \\
Pathology & \\
Tymo carcinoma & $20.0 \pm 5.6^{*}$ \\
Invasive ductal carcinoma & \\
Invasive lobular carcinoma & $8(80.0)$ \\
\hline
\end{tabular}

*Analyzed without the patient who refused surgery

\section{Treatment results}

No visual differences were observed between contrastenhanced MRI before and after MR-HIFU ablation. In patients in whom valid thermometry data were acquired $(\mathrm{n}=7)$, the average maximum temperature of therapeutic sonications was $51.4 \pm 5.7{ }^{\circ} \mathrm{C}$ (range $40.4-61.4{ }^{\circ} \mathrm{C}$ ). Figure 2 shows an example of MR thermometry images during a sonication.
For the 33 of 47 performed sonications with adequate MR thermometry data, no relationship was found between the duration or applied power of the sonications and the temperature increase. In addition, no correlation was found between the product of duration and power (i.e. the applied energy) and the temperature increase (Fig. 3). In particular between different patients, the acoustic powers required to achieve a certain increase in temperature varied considerably. For example, the maximum temperature in patient four was about $59{ }^{\circ} \mathrm{C}$ during $50-\mathrm{W}$ sonications, whereas the maximum temperature in patient eight remained below $55^{\circ} \mathrm{C}$ during three $80-\mathrm{W}$ sonications. Within an individual patient, the peak temperature was more dependent on the applied acoustic power, e.g. for increasing powers, higher maximum temperatures were observed.

The maximum diameter of the area with a temperature higher than $55^{\circ} \mathrm{C}$ varied between 3 and $15 \mathrm{~mm}$. In patient six, no temperatures above $55^{\circ} \mathrm{C}$ were observed on the coronal MR thermometry slice. In contrast, a maximum temperature of $58.5^{\circ} \mathrm{C}$ was measured during the second $70-\mathrm{W}$ sonication in the sagittal slice. In patient nine, a mild temperature increase of about $1 \mathrm{~cm}$ anterior of the focal point was measured, whereas no temperature data were acquired in the actual focal point.

\section{Histopathology}

In six of ten patients, tumour necrosis was observed after MRHIFU ablation by the presence of tissue coagulation and leakage of erythrocytes at H\&E staining (Fig. 4). The maximum diameter of tumour necrosis varied from 3 to $11 \mathrm{~mm}$ (Table 4). Patient four refused to undergo surgery. In patients one, seven and nine, no tumour necrosis was observed. In patient one, only one therapeutic sonication was performed, which was aborted shortly after its initiation. In patient seven, sonications were mainly located in the adipose tissue anterior to the tumour because the tumour eventually turned out to be just outside the range of the transducers. No necrosis was observed inside the tumour; however, fat cell necrosis was observed in the adipose tissue anterior to the tumour. Also in patient nine, the focal point was located outside the tumour, which was caused by an incorrect misalignment correction after the test sonication.
Table 2 Time distribution of MRI-guided high-intensity focused ultrasound (MR-HIFU) treatment

\begin{tabular}{ll}
\hline Stage of the procedure & Time in min, mean \pm SD (range) \\
\hline $\begin{array}{l}\text { Positioning on treatment table } \\
\text { (including MR imaging until contrast injection) }\end{array}$ & $25 \pm 10(5-39)$ \\
Pre-treatment imaging from contrast injection to the first (test) sonication & $59 \pm 27(32-106)$ \\
Treatment time (from first to last sonication) & $46 \pm 17(12-75)$ \\
Post-treatment imaging after the last sonication & $14 \pm 3(7-19)$ \\
Overall procedure time & $145 \pm 29(96-210)$ \\
Overall sonication time & $1.7 \pm 0.8(0.3-2.6)$ \\
\hline
\end{tabular}


Table 3 An overview of the sonications, size, power, duration of sonications, maximum temperature and size of the area(s) that reached a temperature higher than $55{ }^{\circ} \mathrm{C}$ for all patients

\begin{tabular}{|c|c|c|c|c|c|c|}
\hline Patient & Sonication & Size $(\mathrm{mm})$ & Power (W) & Duration (s) & Max temp $\left({ }^{\circ} \mathrm{C}\right)$ & Temp $>55^{\circ} \mathrm{C}(\mathrm{mm})$ \\
\hline \multirow[t]{2}{*}{1} & 1 (test) & 3 & 30 & $8.5^{*}$ & $\mathrm{NA}^{\dagger}$ & $\mathrm{NA}^{\dagger}$ \\
\hline & 2 & 6 & 100 & $8.6^{*}$ & & \\
\hline \multirow[t]{6}{*}{2} & 1 (test) & 3 & 30 & $16.1^{*}$ & $\mathrm{NA}^{\dagger}$ & $\mathrm{NA}^{\dagger}$ \\
\hline & 2 & 3 & 70 & $19.7^{*}$ & & \\
\hline & 3 (test) & 3 & 30 & $12.3^{*}$ & & \\
\hline & 4 & 6 & 60 & $14.9^{*}$ & & \\
\hline & 5 & 6 & 50 & 24.6 & & \\
\hline & 6 & 6 & 70 & $22.1 *$ & & \\
\hline \multirow[t]{2}{*}{3} & 1 (test) & 3 & 40 & $12.9^{*}$ & 56.1 & $10 \times 7$ \\
\hline & 2 & 6 & 70 & $16.8^{*}$ & 52.6 & $3 \times 3$ \\
\hline \multirow[t]{4}{*}{4} & 1 (test) & 3 & 40 & 20.2 & 55.7 & $5 \times 5$ \\
\hline & 2 & 6 & 50 & 24.6 & 59.0 & $3 \times 2$ \\
\hline & 3 & 6 & 50 & 24.6 & 58.3 & $7 \times 5$ \\
\hline & 4 & 6 & 50 & 24.6 & 59.1 & $5 \times 3$ \\
\hline \multirow[t]{7}{*}{5} & 1 (test) & 3 & 40 & 20.0 & $\mathrm{NA}^{\ddagger}$ & $\mathrm{NA}^{*}$ \\
\hline & 2 (test) & 3 & 40 & 20.0 & $\mathrm{NA}^{\ddagger}$ & $\mathrm{NA}^{\ddagger}$ \\
\hline & 3 (test) & 3 & 40 & 20.0 & 55.7 & $8 \times 7$ \\
\hline & 4 (test) & 3 & 30 & 20.0 & 51.1 & No \\
\hline & 5 & 6 & 50 & 24.5 & 61.4 & $15 \times 12$ \\
\hline & 6 & 6 & 60 & $23.2^{*}$ & 57.9 & $12 \times 10$ \\
\hline & 7 & 6 & 50 & 24.5 & 56.4 & $8 \times 7$ \\
\hline \multirow[t]{5}{*}{6} & 1 (test) & 3 & 30 & 20.1 & 45.8 & No \\
\hline & 2 & 6 & 50 & 24.6 & 49.4 & No \\
\hline & 3 & 6 & 60 & 24.6 & 50.3 & No \\
\hline & 4 & 6 & 70 & 24.6 & 52.7 & No \\
\hline & 5 & 6 & 70 & 24.6 & 51.6 & No \\
\hline \multirow[t]{4}{*}{7} & 1 (test) & 3 & 30 & 20.1 & $\mathrm{NA}^{\S}$ & $\mathrm{NA}^{\S}$ \\
\hline & 2 & 3 & 50 & 20.1 & & \\
\hline & 3 & 6 & 70 & 24.6 & & \\
\hline & 4 & 6 & 90 & 24.6 & & \\
\hline \multirow[t]{6}{*}{8} & 1 (test) & 3 & 30 & 20.1 & 43.9 & No \\
\hline & 2 & 6 & 60 & 24.6 & 48.0 & No \\
\hline & 3 & 6 & 80 & 24.6 & 46.8 & No \\
\hline & 4 & 6 & 60 & 24.6 & 49.1 & No \\
\hline & 5 & 6 & 80 & 24.6 & 48.8 & $7 \times 5$ \\
\hline & 6 & 6 & 80 & 24.6 & 51.9 & $5 \times 2$ \\
\hline \multirow[t]{6}{*}{9} & 1 (test) & 3 & 40 & 20.1 & 43.2 & No \\
\hline & 2 & 6 & 60 & 24.6 & 42.5 & No \\
\hline & 3 & 6 & 80 & 24.6 & 42.7 & No \\
\hline & 4 & 6 & 80 & 24.6 & 40.4 & No \\
\hline & 5 (test) & 3 & 40 & 20.1 & 42.7 & No \\
\hline & 6 & 6 & 80 & 24.6 & 46.7 & No \\
\hline \multirow[t]{5}{*}{10} & 1 (test) & 3 & 30 & 20.1 & 45.5 & No \\
\hline & 2 (test) & 3 & 40 & 20.1 & 46.6 & No \\
\hline & 3 (test) & 3 & 40 & 20.1 & 44.0 & No \\
\hline & 4 & 6 & 80 & 24.6 & 54.4 & No \\
\hline & 5 & 6 & 80 & 24.6 & 51.7 & $7 \times 7$ \\
\hline
\end{tabular}

NA not available

* Sonication was prematurely terminated due to an incorrect excessive heating abort

$\dagger$ The quality of the thermometry data was too low for any valid temperature estimates

* During the first two sonications in this patient, the fat signal was not suppressed during RPFS thermometry. No valid thermometry data were acquired

$\S$ Sonications were mainly located in the adipose tissue anterior of the tumour and no valid thermometry data were acquired 

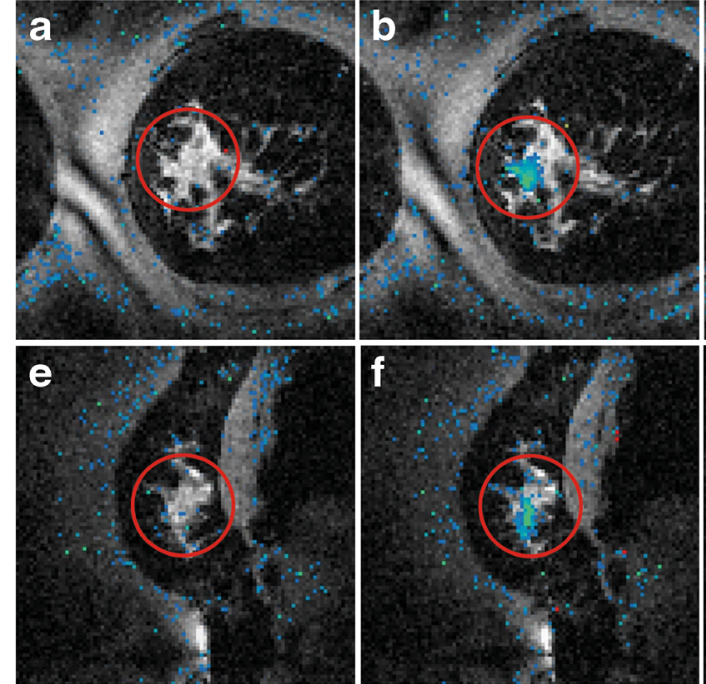

Fig. 2 Magnitude images (grey scale) overlaid with MR thermometry data (colour-coded) during the seventh sonication in patient five; a $50-\mathrm{W}$ sonication with a duration of $24.5 \mathrm{~s}$. The maximum temperature reached

Sonications were performed at 19 different locations: one to four different locations per patient. In the six patients with tumour necrosis, the number of targeted locations was equal to the number of areas with tumour necrosis at histopathology. In these patients, sonications were performed at 13 different locations, corresponding to 13 different areas of tumour necrosis.

\section{Correlation between sonication parameters and the size of tumour necrosis at histopathology}

For these analyses, patients four (no histopathology available), seven and nine (sonications located in adipose tissue) were excluded, yielding 14 different locations. A medium correlation
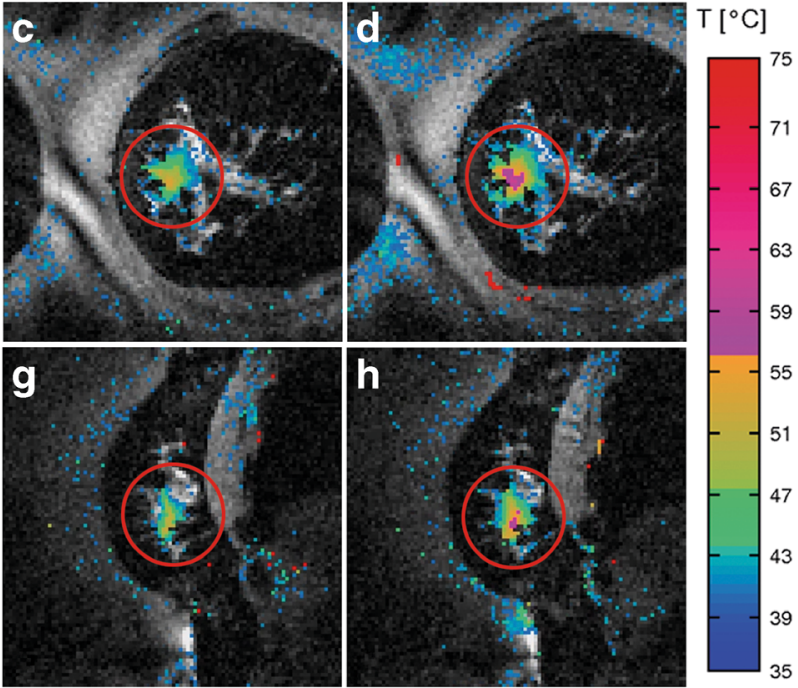

during this sonication was $56.4^{\circ} \mathrm{C}$. Figures a-d and e-h show the coronal and sagittal images through the focal point, respectively, which were acquired with a temporal resolution of $2.25 \mathrm{~s}$

was found between the duration of sonications $(r=0.73$, $\mathrm{p}=0.003)$ and the applied acoustic power $(\mathrm{r}=0.62 \mathrm{p}=0.019)$, and the size of tumour necrosis at histopathology. Furthermore, a good correlation was found between the applied energy and the size of tumour necrosis at histopathology $(r=0.76$, $\mathrm{p}=0.002$, Fig. 5 ). For 11 locations, adequate MR thermometry data were available. No relationship was found between the temperature increase and the size of tumour necrosis at histopathology. Furthermore, no correlation was found between the size of the area with a temperature higher than $55^{\circ} \mathrm{C}$ and the size of tumour necrosis at histopathology. The product of the duration and the temperature increase and the size of tumour necrosis showed a medium correlation $(r=0.74, p=0.01)$.
Fig. 3 Product of the duration (in seconds) and the applied power (in Watts) of the performed sonications (i.e. the applied energy) versus the increase in temperature (in ${ }^{\circ} \mathrm{C}$ ) as measured with MR thermometry

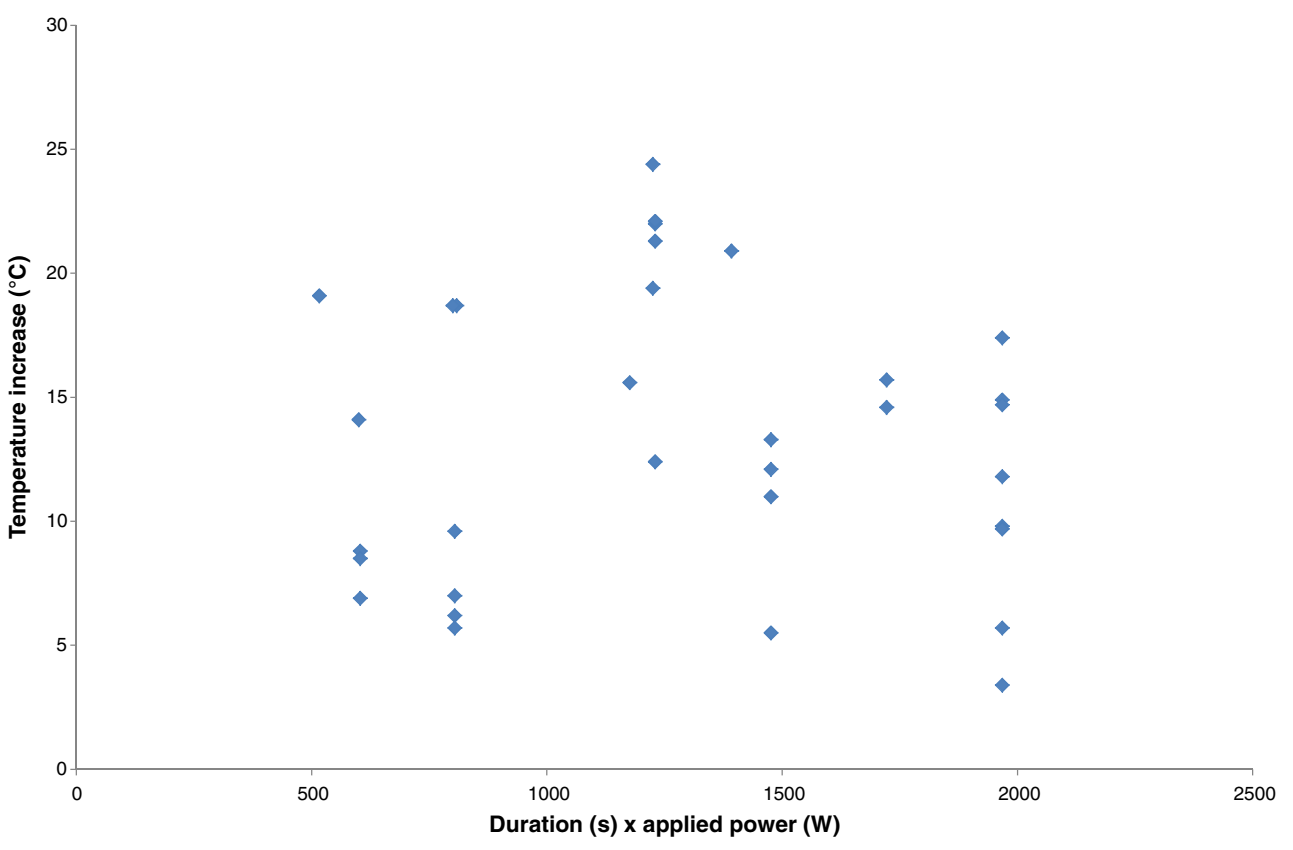


Fig. 4 Macroscopic (a) and microscopic pictures (b-d) of the surgical specimen of the fifth patient. (a) The yellow tissue is adipose tissue and the white tissue is the tumour tissue. The redbrown area inside the tumour indicates the presence of a haemorrhagic area which is caused by MRI-guided high-intensity focused ultrasound (MRHIFU) ablation. (b-d)

Haematoxylin and eosin (H\&E) stainings with increasing magnification. The blue line delineates the invasive tumour which is surrounded by normal fibroglandular tissue and adipose tissue. The area of tumour necrosis is encircled by a black line

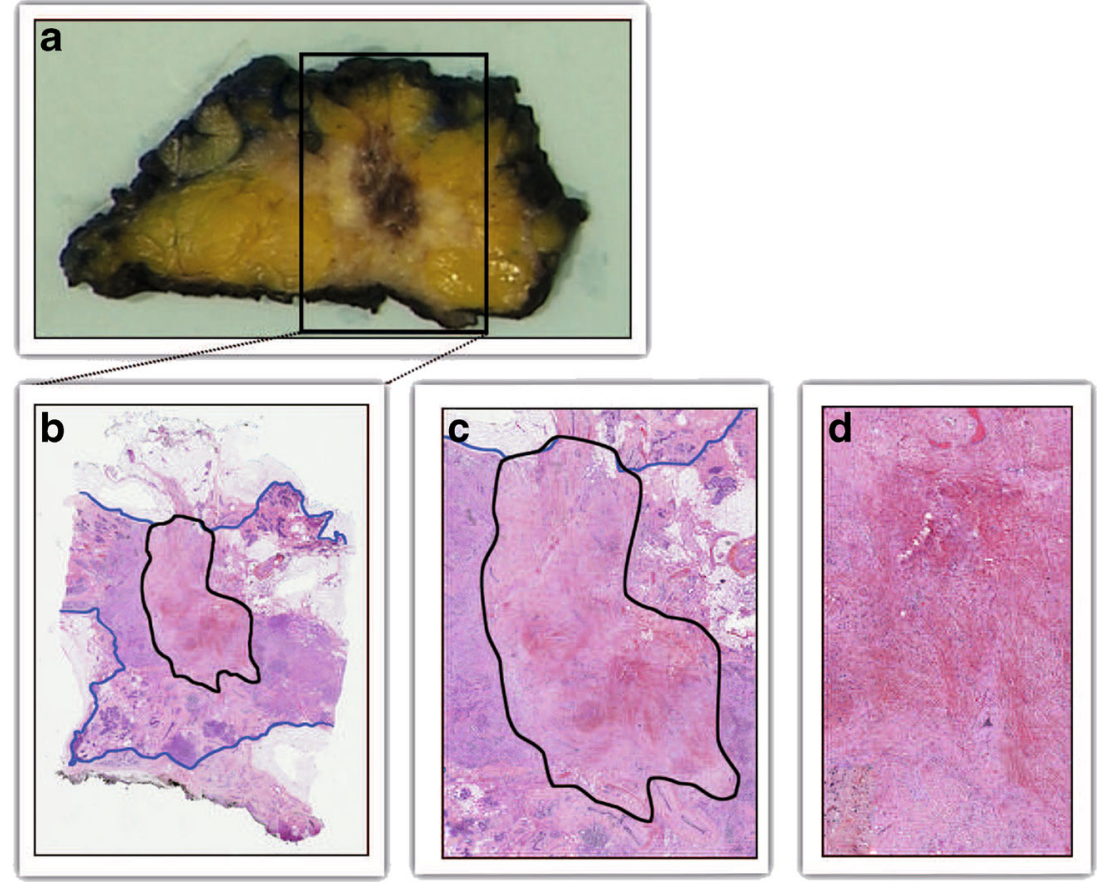

\section{Discussion}

Our results show that MR-HIFU ablation in breast cancer patients with the dedicated breast system is safe and feasible. In none of the patients was skin redness or burns observed. A good correlation was found between the product of duration and power of sonications (i.e. the applied energy) and the size of tumour necrosis at histopathology $(r=0.76, p=0.002)$. Furthermore, in the patients with tumour necrosis at histopathology, the number of targeted locations was equal to the number of areas with tumour necrosis.

No relationship was found between the applied energy and the increase in temperature. Particularly between different patients, we observed that the acoustic powers required to achieve a certain increase in temperature varied considerably. This may be explained by differences in tumour perfusion and patient characteristics, for example breast size or the ratio between glandular and adipose tissue. Another factor influencing the extent of temperature increase is the distance between tumour and ultrasound transducers. In our system, the circular arc of transducers covers $270^{\circ}$. If the targeted area is close to the 'open' part of the arc of transducers, higher powers may be needed to achieve the same increase in temperature. Lastly, the measured temperatures are largely dependent on the position of the MR thermometry slices. During certain treatments (e.g. patients six and nine), the measured temperature increase was not as high as one would expect based on other treatments (e.g. patients four and five). This was caused by an incorrect misalignment correction after the test sonication due to the limited experience of the operator, whereby the MR thermometry slice was not positioned exactly through the focal point during the therapeutic sonications.

No relationship was found between the temperature increase and the size of tumour necrosis or the size of the area with a temperature higher than $55^{\circ} \mathrm{C}$ and the size of tumour necrosis at histopathology. This may be explained by the reasons given before. A medium correlation was found between the product of the duration of sonications and the temperature increase, and the size of tumour necrosis at histopathology. This result, however, has to be considered carefully because it is a very simplified estimation of the complicated relationship between time and temperature [23]. Unfortunately, we were not able to calculate the exact thermal dose with our data. Another limitation of our analyses is that pathology specimens were not reconstructed in 3D. This means that the maximum diameter used as the outcome measure for the size of tumour necrosis is only an estimate of the whole area of tumour necrosis.

This study was designed to investigate the safety and feasibility of the dedicated MR-HIFU breast platform. Therefore, we chose to allow patients with tumours between 3 and $5 \mathrm{~cm}$ in size to participate in this study. Patients with large T2 tumours, however, are generally not appropriate candidates for treatment with minimally invasive ablation techniques such as HIFU [17, 24]. In addition, the partial ablation design of our study is different to that of previous studies. In our opinion, this design is more suitable for investigating safety, e.g. through the possibility of analyzing the location and size of separate sonications. In addition, the relationship between different sonication parameters and the size of tumour necrosis at 
Table 4 An overview of the sonications, locations and tumour necrosis for all patients

\begin{tabular}{llll}
\hline Patient & Sonication & Location & Tumour necrosis (mm) \\
\hline 1 & $1-2$ & 1.1 & No \\
2 & $1-2$ & 2.1 & $3 \times 1$ \\
& $3-6$ & 2.2 & $7 \times 3$ \\
3 & 1 & 3.1 & $5 \times 2$ \\
& 2 & 3.2 & $6 \times 4^{*}$ \\
4 & $1-4$ & 4.1 & $\mathrm{NA}^{\dagger}$ \\
5 & $1-3$ & 5.1 & $7 \times 6$ \\
& $4-7$ & 5.2 & $10 \times 5$ \\
6 & $1-5$ & 6.1 & $11 \times 7^{\S}$ \\
7 & $1-4$ & 7.1 & No \\
8 & $1-3$ & 8.1 & $8 \times 3$ \\
& 4 & 8.2 & $4 \times 3$ \\
& 5 & 8.3 & $9 \times 5$ \\
& 6 & 8.4 & $7 \times 4$ \\
9 & $1-3$ & 9.1 & No \\
& 4 & 9.2 & No \\
& $5-6$ & 9.3 & No \\
& $1-4$ & 10.1 & $9 \times 4$ \\
& 5 & 10.2 & $7 \times 3$ \\
\hline & & &
\end{tabular}

$N A$ not available

* The thermal damage is for the major part present in the glandular tissue outside the tumour due to movement of the patient after the test sonication

$\dagger$ No pathology results available

$\$$ Treatment cells were positioned next to each other and not exactly at the same location

$\S$ Two other small areas of tumour necrosis were observed

No necrosis was observed inside the tumour; however, fat cell necrosis was observed in the adipose tissue surrounding the tumour

histopathology could be analyzed for all different locations. Finally, the partial ablation approach allowed us to compare viable versus ablated tumour tissue at histopathology and the non-ablated tumour tissue could be used for the decision about adjuvant treatments.

One of the shortcomings of MR-HIFU ablation in general is the long duration of the procedure [25]. In our study, the overall procedure time was on average $145 \mathrm{~min}$, while the actual sonication time was only $1.7 \mathrm{~min}$. The most timeconsuming aspects of the treatment procedure were the waiting time after contrast injection, filling of the LUT before every sonication and the delays caused by the need to find a proper navigator signal for MR thermometry. In future studies, the ratio between the actual sonication time and the overall procedure time has to be changed. We chose to treat patients under procedural sedation because of the long duration of treatment in an uncomfortable position. In addition, a regular breathing pattern is preferable for LUT-based corrected MR thermometry. In the first two patients, no valid thermometry data were acquired due to artefacts caused by patient motion and changes in breathing pattern. Consequently, most sonications were prematurely aborted automatically for safety reasons. From patient three onwards, the sedation regimen was changed to esketamine, a sedative agent with minimal impact on respiration. In addition, the sedation specialists gained more experience and gradually increased the dose of analgesics. In patients four to ten, no patient motion or changes in breathing pattern were observed anymore and almost all therapeutic sonications could be fully executed. An additional problem during the first two treatments was the occurrence of ghosting artefacts during MR thermometry, and consequently $\mathrm{MnCl}_{2} \cdot 4 \mathrm{H}_{2} \mathrm{O}$ was added to the water in the breast cup. Thereafter, no such artefacts were observed anymore.

In our study, we did not observe NPVs immediately after MR-HIFU ablation. In other lesions, for example in benign uterine fibroids, it is known that the observed NPV directly after treatment is related to necrosis $[19,26]$. Our results indicate that contrary to other lesions, malignant breast tumours
Fig. 5 Product of the duration (in seconds) and the applied power (in Watts) of the performed sonications (i.e. the applied energy) versus the size of tumour necrosis (in $\mathrm{mm}^{2}$ ) at histopathology

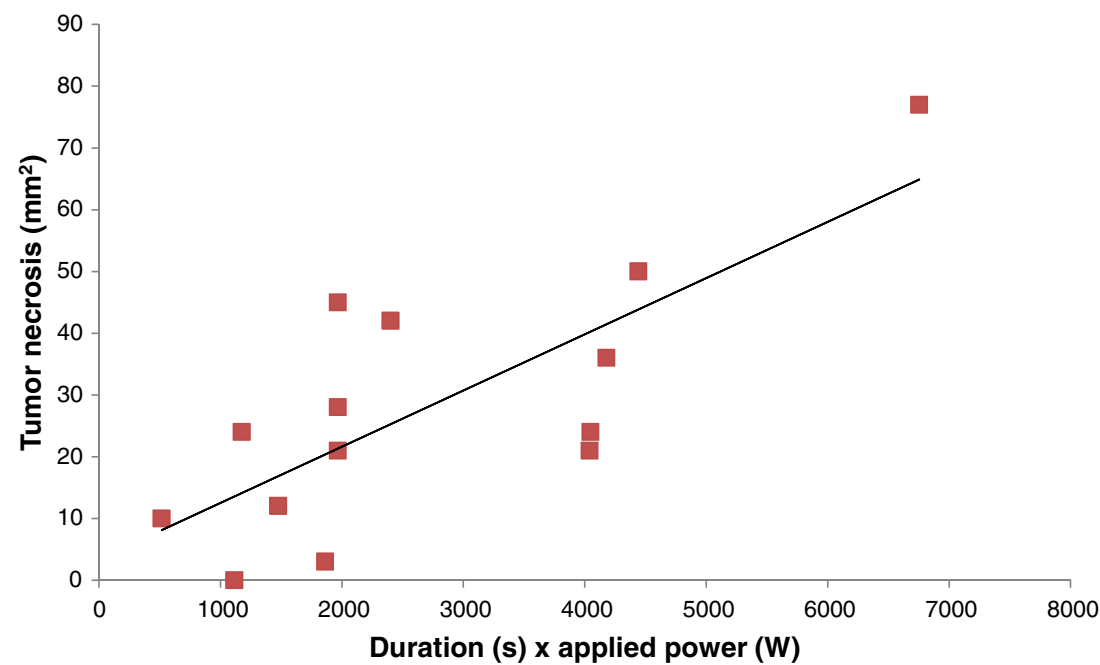


may show slow enhancement directly after MR-HIFU ablation even when there is no evidence for residual tumour at histopathology. This is in agreement with findings reported by previous groups [27] and may be caused by leakage of contrast into the interstitial space after tumour ablation. In addition, we ablated only a small region within a large tumour and no high-temporal resolution DCE-MRI was performed.

In conclusion, we report our first experiences with MR-HIFU ablation of breast cancer using a dedicated breast platform, concluding that MR-HIFU ablation is safe and results in histopathologically proven tumour necrosis.

Acknowledgments The scientific guarantor of this publication is M.A.A.J. van den Bosch. The authors of this manuscript declare relationships with the following company: Philips Healthcare.

One of the authors, Gerald Schubert, is currently employed at Philips Healthcare.

In addition, the MR-HIFU breast platform is developed by Philips Healthcare. This study has received funding from the Center for Translational Molecular Medicine (VOLTA). No complex statistical methods were necessary for this paper. Institutional Review Board approval was obtained by the Institutional Review Board of the University Medical Center Utrecht.

Written informed consent was obtained from all subjects (patients) in this study.

The same patient population is included in Deckers R, Merckel LG, Denis de Senneville B, et al. (2015) Performance analysis of a dedicated breast MR-HIFU system for tumor ablation in breast cancer patients. Phys Med Biol 60:5527-5542

The paper by Decker et al. focuses entirely on the technical aspects of MR-HIFU ablation by analyzing the MR thermometry data in detail, whereas this article is substantially different because it focuses on the clinical outcome with adverse events and outcomes of histopathology.

Methodology: prospective, experimental study.

Open Access This article is distributed under the terms of the Creative Commons Attribution-NonCommercial 4.0 International License (http:// creativecommons.org/licenses/by-nc/4.0/), which permits any noncommercial use, distribution, and reproduction in any medium, provided you give appropriate credit to the original author(s) and the source, provide a link to the Creative Commons license, and indicate if changes were made.

\section{References}

1. Ferlay J, Héry C, Autier P, Sankaranarayanan R (2010) Global burden of breast cancer. Breast Cancer Epidemiology. Springer, New York, pp 1-19

2. Walters S, Maringe C, Butler J et al (2013) Breast cancer survival and stage at diagnosis in Australia, Canada, Denmark, Norway, Sweden and the UK, 2000-2007: a population-based study. Br J Cancer 108:1195-1208

3. Veronesi U, Cascinelli N, Mariani L et al (2002) Twenty-year follow-up of a randomized study comparing breast-conserving surgery with radical mastectomy for early breast cancer. N Engl J Med 347: $1227-1232$

4. Fisher B, Anderson S, Bryant J et al (2002) Twenty-year follow-up of a randomized trial comparing total mastectomy, lumpectomy, and lumpectomy plus irradiation for the treatment of invasive breast cancer. N Engl J Med 347:1233-1241
5. Roubidoux MA, Sabel MS, Bailey JE et al (2004) Small $(<2.0-\mathrm{cm})$ breast cancers: mammographic and US findings at US-guided cryoablation-initial experience. Radiology 233:857-867

6. van den Bosch M, Daniel B, Rieke V et al (2008) MRI-guided radiofrequency ablation of breast cancer: preliminary clinical experience. J Magn Reson Imaging JMRI 27:204-208

7. Zhou W, Zha X, Liu X et al (2012) US-guided percutaneous microwave coagulation of small breast cancers: a clinical study. Radiology 263:364-373

8. Kennedy JE, Ter Haar GR, Cranston D (2003) High intensity focused ultrasound: surgery of the future? Br J Radiol 76:590-599

9. Hynynen K, Darkazanli A, Unger E, Schenck JF (1993) MRIguided noninvasive ultrasound surgery. Med Phys 20:107-115

10. Quesson B, de Zwart JA, Moonen CT (2000) Magnetic resonance temperature imaging for guidance of thermotherapy. J Magn Reson Imaging JMRI 12:525-533

11. Rieke V, Butts Pauly K (2008) MR thermometry. J Magn Reson Imaging JMRI 27:376-390

12. Huber PE, Jenne JW, Rastert R et al (2001) A new noninvasive approach in breast cancer therapy using magnetic resonance imagingguided focused ultrasound surgery. Cancer Res 61:8441-8447

13. Furusawa H, Namba K, Thomsen S et al (2006) Magnetic resonance-guided focused ultrasound surgery of breast cancer: reliability and effectiveness. J Am Coll Surg 203:54-63

14. Furusawa $\mathrm{H}$, Namba K, Nakahara $\mathrm{H}$ et al (2007) The evolving nonsurgical ablation of breast cancer: MR guided focused ultrasound (MRgFUS). Breast Cancer 14:55-58

15. Gianfelice D, Khiat A, Amara M et al (2003) MR imaging-guided focused US ablation of breast cancer: histopathologic assessment of effectiveness- initial experience. Radiology 227:849-855

16. Zippel DB, Papa MZ (2005) The use of MR imaging guided focused ultrasound in breast cancer patients; a preliminary phase one study and review. Breast Cancer 12:32-38

17. Merckel LG, Bartels LW, Köhler MO et al (2013) MR-guided highintensity focused ultrasound ablation of breast cancer with a dedicated breast platform. Cardiovasc Intervent Radiol 36:292-301

18. Köhler MO, Mougenot C, Quesson B et al (2009) Volumetric HIFU ablation under 3D guidance of rapid MRI thermometry. Med Phys 36:3521-3535

19. Voogt MJ, Trillaud H, Kim YS et al (2012) Volumetric feedback ablation of uterine fibroids using magnetic resonance-guided high intensity focused ultrasound therapy. Eur Radiol 22:411-417

20. Deckers R, Merckel LG, Denis de Senneville B et al (2015) Performance analysis of a dedicated breast MR-HIFU system for tumor ablation in breast cancer patients. Phys Med Biol 60:55275542

21. Hey S, Maclair G, de Senneville BD et al (2009) Online correction of respiratory-induced field disturbances for continuous MRthermometry in the breast. Magn Reson Med 61:1494-1499

22. Williamson A, Hoggart B (2005) Pain: a review of three commonly used pain rating scales. J Clin Nurs 14:798-804

23. Sapareto SA, Dewey WC (1984) Thermal dose determination in cancer therapy. Int J Radiat Oncol Biol Phys 10:787-800

24. Merckel LG, van den Bosch MAAJ (2012) Imaging-guided breast cancer ablation. Radiology 265:322-323, author reply 323

25. Kim Y, Keserci B, Partanen A et al (2012) Volumetric MR-HIFU ablation of uterine fibroids: role of treatment cell size in the improvement of energy efficiency. Eur J Radiol 81:3652-3659

26. McDannold N, Tempany CM, Fennessy FM et al (2006) Uterine leiomyomas: MR imaging-based thermometry and thermal dosimetry during focused ultrasound thermal ablation. Radiology 240: 263-272

27. Khiat A, Gianfelice D, Amara M, Boulanger Y (2006) Influence of post-treatment delay on the evaluation of the response to focused ultrasound surgery of breast cancer by dynamic contrast enhanced MRI. Br J Radiol 79:308-314 